\title{
Hypercomplex structures on Kähler manifolds
}

\author{
Misha Verbitsky ${ }^{1}$ \\ verbit@maths.gla.ac.uk, verbit@mccme.ru
}

\begin{abstract}
Let $(M, I)$ be a compact Kähler manifold admitting a hypercomplex structure $(M, I, J, K)$. We show that $(M, I, J, K)$ admits a natural HKT-metric. This is used to construct a holomorphic symplectic form on $(M, I)$.
\end{abstract}

\section{Contents}

1 Introduction 1

1.1 Hvpercomplex manifolds . . . . . . . . . . . . . . . . . . . . . . 1

1.2 HKT metrics and the canonical class . . . . . . . . . . . . . . . 3

2 Calabi-Yau theorem and triviality of canonical bundle 5

$\begin{array}{lll}3 & \text { Kähler metrics and HKT metrics } & 6\end{array}$

$\begin{array}{lll}4 & \text { Supersymmetry on HKT-manifolds with trivial canonical class } & 8\end{array}$

\section{Introduction}

\subsection{Hypercomplex manifolds}

Let $(M, I, J, K)$ be a manifold equipped with an action of the quaternion algebra $\mathbb{H}$ on $T M$. The manifold $M$ is called hypercomplex if the operators $I, J, K \in \mathbb{H}$ define integrable complex structures on $M$. As Obata proved ( $[\mathrm{Ob}]$ ), this condition is satisfied if and only if $M$ admits a torsion-free connection $\nabla$ preserving the quaternionic action:

$$
\nabla I=\nabla J=\nabla K=0 .
$$

Such a connection is called an Obata connection on $(M, I, J, K)$. It is necessarily unique $([\mathrm{Ob}])$.

\footnotetext{
${ }^{1}$ The author is supported by EPSRC grant GR/R77773/01 and CRDF grant RM12354-MO02
} 
Hypercomplex manifolds were defined by C.P.Boyer ([Bo] $)$, who gave a classification of compact hypercomplex manifolds for $\operatorname{dim}_{\mathbb{H}} M=1$.

If the Obata connection $\nabla$, in addition, preserves a quaternionic Hermitian $^{1}$ metric $g$ on $M$, then $(M, I, J, K, g)$ is called hyperkähler. This definition is equivalent to the standard one, see e.g. [Bes].

It is unknown precisely which complex manifold admit hypercomplex structures.

Question 1.1: Consider a compact complex manifold $(M, I)$. Describe the set of hypercomplex structures $(I, J, K)$ compatible with the given complex structure on $M$.

A similar question about hyperkähler structures is easily answered by the Calabi-Yau theorem. Recall that a hyperkähler manifold is holomorphically symplectic. Indeed, consider the 2 -forms $\omega_{J}(\cdot, \cdot)=g(J \cdot, \cdot), \omega_{K}(\cdot, \cdot)=$ $g(K \cdot, \cdot)$; then

$$
\Omega:=\omega_{J}+\sqrt{-1} \omega_{K}
$$

is a nowhere degenerate holomorphic $(2,0)$-form on $(M, I)$ (Bes $)$. A converse result is implied by Calabi-Yau theorem: a holomorphically symplectic compact Kähler manifold is necessarily hyperkähler.

Theorem 1.2: Let $(M, I)$ be a compact holomorphically symplectic manifold with a Kähler form $\omega$. Then there exists a unique hyperkähler metric $g$ on $M$, with the same Kähler class as $\omega$.

Proof: See Bes].

We have no similar description of complex manifolds admitting a hypercomplex structure. In this paper we study the following problem.

Question 1.3: Let $(M, I)$ be a compact complex manifold of Kähler type ${ }^{2}$. When $(M, I)$ admits a hypercomplex structure?

\footnotetext{
${ }^{1} \mathrm{~A}$ metric $b$ is called quaternionic Hermitian if

$$
g(I x, I y)=g(J x, J y)=g(K x, K y)=g(x, y)
$$

for all $x, y \in T M$.

${ }^{2}$ That is, admitting a Kähler metric.
} 
The following theorem gives an answer.

Theorem 1.4: Let $(M, I, J, K)$ be a compact hypercomplex manifold. Assume that $(M, I)$ admits a Kähler structure. Then $(M, I)$ is holomorphically symplectic.

Proof: In Subsection 1.2 we deduce Theorem 1.4 from Theorem 1.9 Theorem 1.10 and Theorem 1.11, which are proven in Sections 2, 3] and 4.

Remark 1.5: By Calabi-Yau theorem (Theorem 1.2), a holomorphically symplectic manifold admits a hyperkähler structure. However, the hypercomplex structure $(M, I, J, K)$ on $M$ can a priori have a different nature. The manifold $(M, I, J, K)$ is hyperkähler if and only if the Obata connection $\nabla$ preserves a metric. However, if the holonomy of $\nabla$ is non-unitarian, such a metric does not exist.

Definition 1.6: Let $(M, I)$ be a compact holomorphically symplectic Kähler manifold, and $(M, I, J, K)$ a hypercomplex structure on $(M, I)$. Then $(M, I, J, K)$ is called exotic if $(M, I, J, K)$ is not hyperkähler, that is, if the holonomy of its Obata connection is not unitarian.

We conjecture that exotic hypercomplex structures do not exist.

\subsection{HKT metrics and the canonical class}

Let $M$ be a hypercomplex manifold. A "hyperkähler with torsion" (HKT) metric on $M$ is a special kind of a quaternionic Hermitian metric, which became increasingly important in mathematics and physics for the last 7 years.

HKT-metrics were introduced by P.S.Howe and G.Papadopoulos ([HP $]$ ) and much discussed in physics literature since then. For an excellent survey of these works written from a mathematician's point of view, the reader is referred to the paper of G. Grantcharov and Y. S. Poon GP.

The term "hyperkähler metric with torsion" is actually misleading, because an HKT-metric is not hyperkähler. This is why we prefer to use the abbreviation "HKT-manifold".

Let $(M, I, J, K)$ be a hypercomplex manifold, $g$ a quaternionic Hermitian form, and $\Omega$ the (2,0)-form on $(M, I)$ constructed from $g$ as in (1.1). The hyperkähler condition can be written down as $d \Omega=0$ ([Bes]). The HKT condition is weaker: 
Definition 1.7: A quaternionic Hermitian metric is called an HKTmetric if

$$
\partial(\Omega)=0
$$

where $\partial: \Lambda_{I}^{2,0}(M) \longrightarrow \Lambda_{I}^{3,0}(M)$ is the Dolbeault differential on $(M, I)$, and $\Omega$ the $(2,0)$-form on $(M, I)$ constructed from $g$ as in (1.1).

It was shown in $\mathrm{HP}$, GP, that this condition is in fact independent from the choice of the triple of complex structures $(I, J, K), I J=-J I=K$ in $\mathbb{H}$. In particular, we could replace the hypercomplex structure $(M, I, J, K)$ with $(M, J, K, I)$. We obtain the following trivial claim

Claim 1.8: Let $(M, I, J, K)$ be a hypercomplex manifold, $g$ a quaternionic Hermitian metric. Consider $g$ as a quaternionic Hermitian metric on a hypercomplex manifold $(M, J, K, I)$. Then $g$ satisfies the HKT-condition on $(M, I, J, K)$ if and only if $g$ satisfies the HKT-condition on $(M, J, K, I)$.

HKT-metrics play in hypercomplex geometry the same role as the Kähler metrics play in complex geometry ( $[\mathrm{V} 1])$.

The proof of Theorem 1.4 is split onto three steps, as follows.

Theorem 1.9: Let $(M, I, J, K)$ be a compact hypercomplex manifold. Assume that $(M, I)$ admits a Kähler structure. Then the ge exists a finite non-ramified covering $\widetilde{M} \longrightarrow M$ such that the canonical bundle of $(\widetilde{M}, I)$ is trivial as a holomorphic vector bundle.

\section{Proof: See Section 2,}

Theorem 1.10: Let $(M, I, J, K)$ be a hypercomplex manifold. Assume that $(M, I)$ admits a Kähler metric $g$. Then $(M, I, J, K)$ admits an HKTmetric $g_{1}$. Moreover, $g_{1}$ can be obtained by averaging $g$ with the $S U(2)$ action induced by quaternions.

Proof: See Section 3 ,

Theorem 1.11: Let $(M, I, J, K)$ be a compact hypercomplex manifold admitting an HKT-metric. Assume that $(M, I)$ admits a Kähler structure. 
Assume, moreover, that there exists a finite non-ramified cover $\widetilde{M} \longrightarrow M$ such that the canonical bundle $K(\widetilde{M}, I)$ has a holomorphic trivialization. Then $(M, I)$ is holomorphically symplectic.

\section{Proof: See Section 4}

Theorem 1.11 concludes the proof of Theorem 1.4 Indeed, consider a compact hypercomplex manifold $(M, I, J, K)$, and assume that $(M, I)$ admits a Kähler metric. By Theorem 1.9 the canonical class of $(M, I)$ is trivial, by Theorem 1.10 $(M, I, J, K)$ is HKT. We arrive at assumptions of Theorem 1.11. obtaining immediately that $(M, I)$ is holomorphically symplectic.

\section{Calabi-Yau theorem and triviality of canonical bundle}

The following proposition is elementary.

Proposition 2.1: Let $(M, I, J, K), \operatorname{dim}_{\mathbb{H}} M=n$ be a hypercomplex manifold, and

$$
c_{1}(M, I) \in H^{2}(M, \mathbb{Z})
$$

the first Chern class of $(M, I)$. Then $c_{1}(M, I)=0$.

Proof: Let $S U(2) \subset \mathbb{H}^{*}$ be the group of unitary quaternions, acting on $T M$. A Riemannian metric $g$ on $M$ is quaternionic Hermitian if and only if $g$ is $S U(2)$-invariant. Taking an arbitrary Riemannian metric and averaging over $S U(2)$, we obtain a quaternionic Hermitian metric. We proved the following trivial claim

Claim 2.2: Let $M$ be a hypercomplex manifold. Then $M$ admits a quaternionic Hermitian metric.

Return to the proof of Proposition 2.1 To show that $c_{1}(M, I)=0$, we need to construct a continuous trivialization of the canonical bundle $K(M, I)=\Lambda^{2 n, 0}(M, I)$, where $2 n=\operatorname{dim}_{\mathbb{C}} M$. Let $g$ be a quaternionic Hermitian metric on $M$, and

$$
\Omega:=g(J \cdot, \cdot)+\sqrt{-1} g(K \cdot, \cdot)
$$


the corresponding non-degenerate $(2,0)$-form on $(M, I)$. Then

$$
\Omega^{n} \in \Lambda_{I}^{2 n, 0}(M)
$$

is a non-degenerate smooth section of the canonical bundle of $\Lambda_{I}^{2 n, 0}(M)=$ $K(M, I)$ of $(M, I)$. Therefore, this bundle is topologically trivial. This gives $c_{1}(M, I)=0$.

The classification of Kähler manifolds with vanishing $c_{1}$ ([Bo, Bea], Bes, ) easily implies the following result.

Theorem 2.3: Let $(M, I)$ be a compact Kähler manifold with

$$
c_{1}(M, I)=0 .
$$

Then there exists a finite non-ramified covering $\widetilde{M} \longrightarrow M$ such that the canonical bundle $K(\widetilde{M}, I)$ is trivial.

Combining Proposition 2.1 and Theorem 2.3, we obtain Theorem 1.9,

Remark 2.4: For a typical non-hyperkaehler compact hypercomplex manifold $(M, I, J, K)$, the complex manifold $(M, I)$ admits no Kähler metrics, and the Calabi-Yau theorem cannot be applied. The canonical bundle $K(M, I)$ is trivial topologically by Proposition 2.1. However, it is in most cases non-trivial as a holomorphic vector bundle, even if one passes to a finite covering. It is possible to show that $K(M, I)$ is non-trivial for all hypercomplex manifold $(M, I, J, K)$ such that $(M, I)$ is a principal toric fibration over a base which has non-trivial canonical class; these include quasiregular Hopf manifolds and semisimple Lie groups with hypercomplex structure constructed by D. Joyce $([\mathbb{J}])$.

\section{Kähler metrics and HKT metrics}

Let $(M, I, J, K)$ be hypercomplex manifold. Since $J$ and $I$ anticommute, $J$ maps $(p, q)$-forms on $(M, I)$ to $(q, p)$-forms:

$$
J: \Lambda_{I}^{p, q}(M) \longrightarrow \Lambda_{I}^{q, p}(M) .
$$

Definition 3.1: Let $\eta \in \Lambda_{I}^{2,0}(M)$ be a $(2,0)$-form on $(M, I)$. Then $\eta$ is called $J$-real if $J(\eta)=\bar{\eta}$, and $J$-positive if for any $x \in T^{1,0}(M, I)$, 
$\eta(x, J(\bar{x})) \geqslant 0$. We say that $\eta$ is strictly $J$-positive if this inequality is strict for all $x \neq 0$.

Denote the space of $J$-real, strictly $J$-positive $(2,0)$-forms by $\Lambda_{>0}^{2,0}(M, I)$.

We need the following linear-algebraic lemma, which is well known (see e.g. V2]).

Lemma 3.2: Let $M$ be a hypercomplex manifold. Then $\Lambda_{>0}^{2,0}(M, I)$ is in one-to-one correspondence with the set of quaternionic Hermitian metrics $g$ on $M$. This correspondence is given by

$$
g \longrightarrow g(J \cdot, \cdot)+\sqrt{-1} g(K \cdot, \cdot),
$$

and the inverse correspondence by

$$
\Omega \longrightarrow g(x, y):=\Omega(x, J(\bar{y})) .
$$

Lemma 3.3: Let $(M, I, J, K)$ be a hypercomplex manifold, $g_{1}$ a Hermitian metric on $(M, J), \omega_{1}=g_{1}(\cdot, J \cdot)$ the corresponding differential 2-form, and $\Omega_{1}$ the $\Lambda_{I}^{2,0}(M)$-part of $\omega_{1}$. Then $\Omega_{1}$ is strictly $J$-positive and $J$-real.

Proof: Since $\omega_{1}$ is a $(1,1)$-form on $(M, J)$, we have $J\left(\omega_{1}\right)=\omega_{1}$. Therefore, $J\left(\Omega_{1}\right)=\bar{\Omega}_{1}$, and $\Omega_{1}$ is $J$-real.

Given $x \in T_{I}^{1,0}(M), x \neq 0$, the number $\Omega_{1}(x, J(\bar{x}))$ is real because $\Omega_{1}$ is $J$-real. On the other hand,

$$
\Omega_{1}(x, J(\bar{x}))=\omega_{1}(x, J(\bar{x}))=g_{1}(x, \bar{x})>0,
$$

because $\Omega_{1}$ is a $(2,0)$-part of $\omega_{1}$ and $x, J(\bar{x})$ are $(1,0)$-vector fields. We have shown that $\Omega_{1}$ is strictly $J$-positive. This proves Lemma 3.3.

We also have the following trivial claim

Claim 3.4: In assumptions of Lemma 3.3, let

$$
\partial: \Lambda_{I}^{p, q}(M) \longrightarrow \Lambda_{I}^{p+1, q}(M)
$$

denote the standard Dolbeault differential $\partial$ on $(M, I)$. Then $\partial \Omega_{1}$ is the $(3,0)$-part of $d \omega_{J}$. In particular, if $g_{1}$ is $\operatorname{Kähler}$ on $(M, J)$ then $\partial \Omega_{1}=0$. 
Proof: By definition, $\Omega_{1}$ is the $(2,0)$-part of $\omega_{J}$, and $\partial \Omega_{1}$ is the $(3,0)$ part of $d \Omega_{1}$.

Remark 3.5: Let $\varphi$ be a Kähler potential for the Kähler form $\omega_{1}$ on $(M, J)$. By Claim 2.3 of [V2], on $(M, I)$ we have $\Omega_{1}=\partial \partial_{J} \varphi$, where $\partial_{J}=$ $-J \circ \bar{\partial} \circ J$. The function $\varphi$ satisfying $\Omega_{1}=\partial \partial_{J} \varphi$ for an HKT-form $\Omega_{1}$ is called an HKT-potential for an HKT-form $\Omega_{1}$.

Now, let $(M, I, J, K)$ be a hypercomplex manifold, and $g_{1}$ a Kähler metric on $(M, J)$. Consider the form $\Omega_{1} \in \Lambda_{I}^{2,0}(M)$ constructed above. Then $\Omega_{1}$ is strictly $J$-positive and $J$-real by Lemma 3.3 and hence corresponds to a quaternionic Hermitian metric $g$ on $(M, I, J, K)$. By Claim 3.4 $\partial \Omega_{1}=0$, hence $g$ is HKT. Doing all calculations explicitly, a reader can show that $g$ is obtained from $g_{1}$ by averaging over $S U(2)$ (we shall not use this claim). This proves Theorem 1.10, Indeed, in assumptions of Theorem 1.10 we are given a Kähler metric on $(M, I)$, so the above argument gives an HKT-metric on the hypercomplex manifold $(M, J, K, I)$; this is equivalent to having an HKT-metric on $(M, I, J, K)$, as Claim 1.8 implies.

\section{Supersymmetry on HKT-manifolds with trivial canonical class}

Let $(M, I, J, K, g)$ be an HKT-manifold, and $K(M, I)$ its canonical class. Using the quaternionic Hermitian metric $g$ we trivialize the canonical class by a smooth non-degenerate section as in Proposition 2.1 Let $K^{1 / 2}$ be the square root of the canonical bundle corresponding to this trivialization. Writing $K(M, I)$ as a trivial bundle with the Chern connection $\nabla_{\text {triv }}+\theta$, we define $K^{1 / 2}$ as a trivial bundle with the conection $\nabla_{\text {triv }}+\frac{1}{2} \theta$. This connection is clearly induced by a holomorphic structure on $K^{1 / 2}$, and $K^{1 / 2} \otimes K^{1 / 2}$ is isomorphic to $K$ as a holomorphic line bundle.

In V1] we proved the following theorem, which is implied by an analogue of the Lefschetz-type $\mathfrak{s l}(2)$-action in the HKT setting.

Theorem 4.1: Let $(M, I, J, K)$ be a compact HKT-manifold, $\operatorname{dim}_{\mathbb{H}} M=$ $n$, and $K^{1 / 2}$ the square root of a canonical bundle $K(M, I)$ constructed as above. Consider the Dolbeault class $[\bar{\Omega}] \in H_{\bar{\partial}}^{0,2}(M, I)=H^{2}\left(\mathcal{O}_{(M, I)}\right)$ of $\bar{\Omega}$, 
where $\Omega \in \Lambda_{I}^{2,}(M)$ is the HKT-form of $M$, and let

$$
H^{l}\left(K^{1 / 2}\right) \stackrel{\wedge[\bar{\Omega}]^{n-l}}{\longrightarrow} H^{2 n-l}\left(K^{1 / 2}\right)
$$

be the corresponding multiplicative map on the holomorphic cohomology of $K^{1 / 2}$. Then (4.1) is an isomorphism.

Proof: In V1] it was shown that the natural operator

$$
L_{\Omega}: H^{l}\left(K^{1 / 2}\right) \longrightarrow H^{l+2}\left(K^{1 / 2}\right)
$$

belongs to an $\mathfrak{s l}(2)$-triple. This is used in V1 to obtain Theorem 4.1 in the same way as one obtains a similar result for the cohomology of a Kähler manifold.

When $K(M, I)$ is a trivial holomorphic bundle, $K^{1 / 2}$ is also a trivial bundle. We obtain that

when $K(M, I)$ is trivial, $[\Omega]^{n}$ is a generator of $H^{2 n}\left(K^{1 / 2}\right) \cong$ $H^{0}\left(K^{1 / 2}\right)^{*}=\mathbb{C}$

(the last isomorphism is provided by the Serre's duality, using the triviality of the canonical bundle). Now we can prove Theorem 1.11

Let $(M, I, J, K)$ be a compact HKT-manifold, with $\widetilde{M}$ a non-ramified finite covering of $M$ with the canonical bundle $K(\widetilde{M}, I)$ trivial. Assume that $(M, I)$ admits a Kähler metric. By Calabi-Yau theorem ( Yau $),(M, I)$ admits a Ricci-flat Kähler metric $h$. Let $\bar{\Omega}_{h} \in \Lambda_{I}^{0,2}(M)$ be a harmonic representative of the cohomology class $[\bar{\Omega}] \in H_{\bar{\partial}}^{0,2}(M, I)$ under $h$. Since $(M, I)$ is Kähler, the harmonic $(2,0)$-form $\Omega_{h}$ is holomorphic. By BochnerLichnerowicz theorem ( $\underline{\mathrm{Bes}})$, this implies

$$
\nabla_{h} \Omega_{h}=0
$$

where $\nabla_{h}$ is the Levi-Civita connection of $h$ (this is true for any holomorphic form $\Omega_{h}$ on a Ricci-flat compact Kähler manifold). Let $\widetilde{\Omega}_{h}, \widetilde{O}$ mega be $\Omega_{h}$, $\Omega$ lifted to $\widetilde{M}$. By (4.2), $\widetilde{\Omega}^{n}$, and hence $\widetilde{\Omega}_{h}^{n}$, represents non-zero class in cohomology of $(\widetilde{M}, I)$. This implies $\Omega_{h}^{n} \neq 0$. By (4.3), we also have $\nabla_{h} \Omega_{h}^{n}=$ 0 , hence $\Omega_{h}^{n}$ trivializes $\Lambda_{I}^{2 n, 0}(M)$. We obtain that $\Omega_{h}$ is a non-degenerate holomorphic symplectic form on $(M, I)$. This proves Theorem 4.1. We finished the proof of Theorem 1.4 
Acknowledgements: This paper appeared as a result of a very rewarding colloboration with S. Alesker. I am also grateful to D. Kaledin and D. Kazhdan for interesting discussions, and to S. Alesker for pointing out errors in the early version of this manuscript.

\section{References}

[Bea] Beauville, A. Varietes Kähleriennes dont la première classe de Chern est nulle. J. Diff. Geom. 18, pp. 755-782 (1983).

[Bes] Besse, A., Einstein Manifolds, Springer-Verlag, New York (1987)

[Bo] Bogomolov, F.A., Hamiltonian Kähler manifolds, Sov. Math., Dokl. 19, 1462-1465 (1978).

[Bo] Boyer, Charles P. A note on hyper-Hermitian four-manifolds. Proc. Amer. Math. Soc. 102 (1988), no. 1, 157-164.

[GP] Grantcharov, G., Poon, Y. S., Geometry of hyper-Kähler connections with torsion, math.DG/9908015 also in Comm. Math. Phys. 213 (2000), no. 1, 19-37.

[GH] Griffiths, Ph., Harris, J., Principles of Algebraic Geometry, Wiley-Intersience, New York, 1978.

[HP] Howe, P. S. Papadopoulos, G., Twistor spaces for hyper-Kähler manifolds with torsion, Phys. Lett. B 379 (1996), no. 1-4, 80-86.

[J] Joyce, Dominic, Compact hypercomplex and quaternionic manifolds, J. Differential Geom. 35 (1992) no. 3, 743-761

[Ob] Obata, M., Affine connections on manifolds with almost complex, quaternionic or Hermitian structure, Jap. J. Math., 26 (1955), 43-79.

[V1] Verbitsky, M., Hyperkähler manifolds with torsion, supersymmetry and Hodge theory, math.AG/0112215 47 pages, also in Asian J. of Math., Vol. 6 (4), pp. 679-712 (2002).

[V2] Verbitsky, M., Hyperkähler manifolds with torsion obtained from hyperholomorphic bundles,, Math. Res. Lett. 10 (2003), no. 4, 501-513, also in math.DG/0303129

[Yau] Yau, S. T., On the Ricci curvature of a compact Kähler manifold and the complex MongeAmpère equation I., Comm. on Pure and Appl. Math. 31, 339-411 (1978).

Misha Verbitsky

University of Glasgow, Department of Mathematics,

15 University Gardens, Glasgow G12 8QW, Scotland,

Institute of Theoretical and Experimental Physics

B. Cheremushinskaya, 25, Moscow, 117259, Russia

verbit@maths.gla.ac.uk, verbit@mccme.ru 